\title{
Mean-Field Equations for Spin Models with Orthogonal Interaction Matrices
}

\author{
Giorgio Parisi and Marc Potters* \\ Dipartimento di Fisica and INFN, Università di Roma La Sapienza \\ P. A. Moro 2, 00185 Roma (Italy)
}

March 1, 1995

\begin{abstract}
We study the metastable states in Ising spin models with orthogonal interaction matrices. We focus on three realizations of this model, the random case and two non-random cases, i.e. the fully-frustrated model on an infinite dimensional hypercube and the so-called sine-model. We use the mean-field (or TAP) equations which we derive by resuming the high-temperature expansion of the Gibbs free energy. In some special non-random cases, we can find the absolute minimum of the free energy. For the random case we compute the average number of solutions to the TAP equations. We find that the configurational entropy (or complexity) is extensive in the range $T_{\mathrm{RSB}}<T<T_{\mathrm{M}}$. Finally we present an apparently unrelated replica calculation which reproduces the analytical expression for the total number of TAP solutions.
\end{abstract}

cond-mat/9503009

*potters@uranus.roma1.infn.it 
The aim of this paper is to study the mean field equations (the TAP equations) for the local magnetization for the fully frustrated Ising model on an hypercube, or equivalently on a single cell of an hypercubic lattice in the limit of infinite dimensions.

The model is very interesting and it has been widely studied. It belongs to the wide class of models which have a non-random Hamiltonian but they behave in a similar (or identical) way to other random models. These models can be studied using the usual techniques for random systems (e.g. the replica method) and in this way one can obtain the correct results (with maybe the exception of the equilibrium behavior at low temperature) [1, 目].

In this model (as in the other models of the same class) there are at least two transitions:

- The dynamical transition at which the correlation time diverges. At this temperature static (i.e. equal time quantities) are fully regular.

- An equilibrium transition at which the replica symmetry is broken. Below this temperature there are many equilibrium states available to the system

If we cool an infinite system below the dynamical transition, its energy does not go to the equilibrium energy and the system remains trapped in a metastable state of higher energy.

Generally speaking one can associate to each stable or metastable state a solution of the TAP equation. On the contrary the inverse relation does not hold. There are many solutions of the TAP equations which do not correspond to stable of metastable states. According to the folklore a TAP solution correspond to a metastable state only if it is separated by other solutions by a barrier whose height diverges with the volume; it seems most of the solutions are separated by other solutions by finite barriers.

The relation between metastable states and the exponentially large persistency time in a given metastable state from one side, and the properties of the solutions of the TAP equations has never fully understood. The aim of this paper contribute to this direction by computing some of the properties of the solutions of the TAP equation in a model which has metastable states.

An other interesting property of the model (absent in its random correspondent) is the presence of a very low energy state, which cannot be reached with continuity coming from the high temperature phase. At a given temperature the system has a first transition to a state with very low energy (the crystal state). The behavior of the system in this low temperature phase may be understood with good precision by considering the corresponding TAP equation.

There is also an other point on which we would like to call the attention of the reader. For the Sherrington-Kirkpatrick (SK) model it has already noticed that the computation of the total number of solutions of the TAP equations turns out to be equivalent to a apparently unrelated replica computation. More precisely it was found that:

$$
\overline{\mathcal{N}} \sim \lim _{m \rightarrow \infty} Z_{m}
$$


where prefactors have been neglected and only the exponentially large terms have been taken into account $Z_{m}$ is the partition function computed by breaking the replica symmetry into two groups of replicas, one with $m$ elements, the other with $n-m$ terms.

The relation is quite surprising, because the r.h.s. may be evaluated without having to write down the very TAP equation.

Apparently there is no known explanation for this phenomenon. Our contribution to this point is to observe that the previous relation holds also in this case, where the form of the TAP equation is much more complex. It is quite like (as also suggested by Cugliandolo and Kurchan), that there should be an isomorphism of two algebraic structure which explain this equality, but we have not done progresses in this direction.

It may be interesting to recall that a similar phenomenon happens in ferromagnetic systems with a random temperature or magnetic field. Let us consider the case of random magnetic field. Here we are interested find the probability of having more than one solution to the stochastic differential equation

$$
-\Delta \phi(x)+m^{2} \phi(x)+g \phi(x)^{3}=h(x)
$$

which plays the role of the TAP equation in this model.

In the replica approach [3, 4, 5] it was found that the existence of many solutions is related to the presence of non trivial saddle points, where the field $\phi_{a}(x)$ for $a=1, n$ $(n=0)$ is of the form

$$
\begin{array}{r}
\phi_{a}(x)=f(x) \text { for } 1 \leq a \leq m \\
\phi_{a}(x)=f(x)+\frac{g(x)}{m} \text { for } m<a \leq n,
\end{array}
$$

and one consider the limit where $m \rightarrow \infty$.

Also in this case the replica symmetry is broken by dividing the replicas into two groups, one with $m$ elements, the other with $n-m$ terms, and taking the limit $m \rightarrow \infty$. This phenomenon seems to be quite widespread.

The plan of the paper is the following.

In section two we recall the definition of three different models, which have the same high temperature expansion: the random orthogonal model (ROM) where the coupling matrix is a random orthogonal matrix, the sine model, where the elements of the coupling matrix can be written as the sine of an appropriate expression, and the fully frustrated models on a single hypercubic cell (FFM). These three models are identical in the high temperature expansion, because they have very similar coupling matrices.

In section three we use the high temperature expansion to derive the TAP equations.

In section four we study the property of the solution of the TAP equation corresponding the lowest lying state (of zero energy) which exists in the sine model and likely exists in the fully frustrated hypercube (the so called crystal state).

In section five we compute the average number of TAP solution for ROM. We study their properties as function of their free energy both at $T=0$ and $T \neq 0$. We also 
find a relation among the properties of the TAP equations and the marginality condition for the dynamical transition and show that the replica symmetric free energy can be written as the sum of contributions from a large number of metastable states above the RSB transition.

In section six we present the computation of the total number of TAP solutions with the two replica group method we have described before.

Finally in the appendix we present some technical details needed in the computations of section five.

\section{The Model}

In what follows we will consider the model defined by the Hamiltonian

$$
H=-\frac{1}{2} \sum_{i j} J_{i j} \sigma_{i} \sigma_{j}
$$

where $\left\{\sigma_{i}\right\}$ is a set of $N$ Ising spin variables $\left(\sigma_{i}= \pm 1\right)$ and $J_{i j}$ is an $N \times N$ symmetric orthogonal matrix with large connectivity $(z \gg 1)$. To lighten the notation, the matrix $J_{i j}$ will be taken to have zeros on the diagonal I].

\subsection{Random Orthogonal Model}

In the random orthogonal model (ROM) the coupling matrix is chosen at random in the set of orthogonal symmetric matrices. The probability distribution (or integration measure) is defined by writing $\mathbf{J}=\mathbf{O D O}^{-\mathbf{1}}$ with $\mathbf{D}$ a diagonal matrix composed of \pm 1 and $\mathbf{O}$ a generic orthogonal matrix (not necessarily symmetric) whose probability distribution is defined by the Haar measure on the orthogonal group. We will make use of the identity [6]

$$
\int \mathcal{D} \mathbf{J} \exp \left\{\operatorname{Tr} \frac{\mathbf{J A}}{2}\right\}=\exp \left\{N \operatorname{Tr} G\left(\frac{\mathbf{A}}{N}\right)\right\},
$$

which holds in the large $N$ limit when $\mathbf{A}$ is a symmetric matrix of finite rank and where $G(x)$ is given by

$$
G(x)=-\frac{1}{4} \log \left(\frac{\sqrt{1+4 x^{2}}+1}{2}\right)+\frac{1}{4} \sqrt{1+4 x^{2}}-\frac{1}{4} .
$$

Notice that integration over matrices chosen from a Gaussian distribution (SK model) also yields Eq. (5) but with $G(x)=x^{2} / 4$.

\footnotetext{
${ }^{1}$ Strictly speaking a generic matrix $\mathbf{J}$ in ROM and the one for the sine model have non-zero diagonal elements, nevertheless the matrix obtained by setting those elements to zero will also be orthogonal in the large $N$ limit.
} 


\subsection{Sine Model}

The coupling matrix for the sine model is given by

$$
J_{i j}=\frac{2}{\sqrt{2 N+1}} \sin \left(\frac{2 \pi i j}{2 N+1}\right) .
$$

The matrix $\mathbf{J}$ is obviously symmetric and its orthogonality follows from orthonormality relations among simple harmonics. This model was introduced in [6] as a simple Hamiltonian that admits a complex ground state for special values of $N$. More precisely, if $2 N+1$ is prime and $N$ is odd then the Legendre sequence

$$
\sigma_{j}=j^{N} \quad(\bmod 2 N+1)
$$

which consists of \pm 1 is a ground state configuration of the sine model with energy density $-1 / 2$. This fact is by no means obvious; the interested reader is referred to [6] for details.

Monte Carlo simulations have shown that the thermodynamical properties of the sine model are the same as that of ROM. The only difference is that for the sine model with $2 N+1$ prime there exists a low-lying state - never seen for large $N$ when cooling from high temperature - in which the system remains (at low temperature) if it is put there by hand. One explanation of the similarity between the sine model and ROM is that Eq. (17) can be viewed as a (bad) pseudo-random generator, and therefore the couplings are, for all practical purpose, random.

\subsection{Fully-Frustrated Model}

Frustration in an Ising-like system (with $J_{i j}= \pm 1$ ) is defined by the product of the couplings over a given plaquette [7]. If this product is -1 the plaquette is said to be frustrated. One can construct a coupling matrix on a $d$ dimensional simple cubic lattice such that every plaquette is frustrated. This construction is not unique but all realizations are gauge equivalent so the thermodynamical properties of the model are unique. For a single hypercubic cell, the fully-frustrated condition imposes [8]

$$
\sum_{k} J_{i k} J_{k j}=d \delta_{i j}
$$

Therefore if we divide the couplings by $\sqrt{d}$ the matrix $\mathbf{J}$ will be orthogonal and symmetric. The thermodynamical limit will be taken by letting $d$ go to infinity, which will also insure that the coordination number is large.

Note that unlike SK on a cubic lattice, for the fully-frustrated model (FFM) the $d \rightarrow \infty$ limit of a single hypercube is not completely equivalent to that of the lattice. The reason is that for SK the distribution of the eigenvalues of the coupling matrix (the celebrated Wigner semi-circle law) is the same both on the single cell and on the lattice. In the fully-frustrated case, the matrix $\mathbf{J}$ has only two eigenvalues $\{-1,1\}$ for the unit 
hypercube, while it has a continuous distribution for the lattice, in the range $[-2,2]$ with rms value $\sqrt{2}$ [9]. Heuristically, in the high-T phase the system is sensitive to all eigenvalues of the matrix: as we shall see, both models (lattice and unit cell) have the same free energy up to a rescaling of $\beta$ by a factor of $\sqrt{2}$. On the other hand, in the low- $T$ phase, it is the largest eigenvalues of $\mathbf{J}$ that matter: for example the ground state of the lattice has twice the energy density of that of the unit cell. It is this difference between 2 and $\sqrt{2}$ that makes it impossible to go from a single cell to the lattice with a simple rescaling of the temperature: their qualitative behavior should nevertheless be the same.

The ground state configuration of FFM is not known for $d>8$. It is conjectured that, with the $\sqrt{d}$ normalization, the ground state energy density goes to $-1 / 2$ for large $d$ and that it is equal to $-1 / 2$ when $d$ is a perfect square. As with the sine model, Monte Carlo simulations of FFM are well described by ROM; furthermore they show aging effects, which strengthens our belief that FFM is a glassy system [10].

\section{Mean-Field Free Energy}

\subsection{Standard High-Temperature Expansion}

Our goal is to derive the TAP equations for the orthogonal model using the hightemperature expansion of the magnetization-dependent (Gibbs) free energy. Before doing so, let us try to understand the standard $\left(m_{i}=0\right)$ high-temperature expansion. We can write

$$
e^{-\beta F}=2^{N} \operatorname{det}^{1 / 2}(\beta \mathbf{J}) \int \prod_{i} \frac{d \phi_{i}}{\sqrt{2 \pi}} \exp \left\{-\frac{1}{2 \beta} \sum_{i j} J_{i j}^{-1} \phi_{i} \phi_{j}+\sum_{i} \log \cosh \left(\phi_{i}+h_{i}\right)\right\},
$$

where we have introduced a site-dependent magnetic field $h_{i}$ for later convenience; in this section we set it to zero. We can view this partition function as a theory of $N$ fields in zero dimension. In this language the high-temperature free energy $F(\beta)$ is the sum of connected diagrams whose vertex factors are the coefficients of the Taylor series for $\log \cosh x$, with the propagator $\beta J_{i j}$. The first few terms are

$$
\begin{aligned}
-\beta F(\beta)= & N \log 2+\frac{\beta^{2}}{4} \sum_{i j} J_{i j}^{2}+\frac{\beta^{3}}{6} \sum_{i j k} J_{i j} J_{j k} J_{k i} \\
& -\frac{\beta^{4}}{4} \sum_{i j k} J_{i j}^{2} J_{j k}^{2}+\frac{\beta^{4}}{12} \sum_{i j} J_{i j}^{4}+\frac{\beta^{4}}{8} \sum_{i j k l} J_{i j} J_{j k} J_{k l} J_{l i}+O\left(\beta^{5}\right),
\end{aligned}
$$

where $J_{i i}=0$ excludes 'petals'-terms involving $\sum J_{i i}$. The only terms that survive the large $N$ limit are the so-called 'cactus' diagrams - trees made out of loops joined at the vertices (see Fig. 1). The sum of $J_{i j}$ 's for cactus diagrams is equal to $N$ if the lengths of all loops are even (even-cacti) and zero otherwise. To show this, we take a 
cactus diagram and contract each loop to a point starting from the outermost loops, the contraction is done using the orthogonality relation

$$
\sum_{k} J_{i k} J_{k j}=\delta_{i j}
$$

In other words, the high-temperature free energy is given by $N$ times the sum over the combinatorial factors and powers of $\beta$ of all even-cactus diagrams. In particular this free energy is independent of the particular choice of the orthogonal matrix $\mathbf{J}$.

There is more than one way to calculate this sum. Since it is independent of the matrix $\mathbf{J}$ it must be equal to the (annealed) average over such matrices, recovering the result of Ref. [6]

$$
-\beta F(\beta)=N \log 2+N G(\beta)
$$

with $G(x)$ given by Eq. (6).

More directly, we notice that the desired diagrams are precisely those summed by the high-temperature series of the spherical model. In the spherical model the Ising variables $\sigma_{i}$ are replaced by continuous variables $S_{i}$ constrained to have $\sum_{i} S_{i}^{2}=N$. Fixing this last constraint with a Lagrange multiplier, the sum over all configurations is then a Gaussian integral which can be done exactly knowing the eigenvalues of the matrix $\mathbf{J}$, i.e., 1 and -1 in equal proportions. After eliminating the Lagrange multiplier, we find

$$
-\beta F_{\mathrm{sph}}(\beta)=\frac{N}{2}(\log 2 \pi+1)+N G(\beta)
$$

with the same $G(x)$. The first terms in Eq. (13) and Eq. (14) are the volume of phase space of the two respective models. A similar analysis was done in Ref. [9] for FFM on the full lattice. They also found Eq. (13) but with $G(\beta)$ replaced with $G(\sqrt{2} \beta)$.

For comparison, the high-temperature series for SK is easily done; the only surviving diagram is the first one in Eq. (11) leaving us with

$$
-\beta F_{\mathrm{SK}}(\beta)=N \log 2+N \frac{\beta^{2}}{4},
$$

where we have used that $\sum J_{i j}^{2}$ is self-averaging.

\subsection{Gibbs Free Energy}

The high-T free energy we have just calculated does not tell us anything about the lowtemperature phase. It does not have any singularity for positive $\beta$ which might signal a phase transition. What we need to compute is the magnetization-dependent free energy (often called the Gibbs free energy). In SK this quantity is exactly the TAP free energy which is still valid in the low-temperature phase. The program is simple, we need to expand Eq. (10) in powers of $\beta$ and $h_{i}$ and perform a Legendre transform, passing from the variables $h_{i}$ to $m_{i}$ using

$$
\Phi\left(\beta, m_{i}\right) \equiv F\left(\beta, h_{i}\right)-\sum_{i} h_{i} m_{i} \quad \text { with } \quad m_{i} \equiv \frac{\partial F}{\partial h_{i}} .
$$


Calculating the first few terms in $\beta$ by hand, we find

$$
\begin{aligned}
-\beta \Phi\left(\beta, m_{i}\right)= & -\frac{1}{2} \sum\left\{\left(1+m_{i}\right) \log \left[\frac{1}{2}\left(1+m_{i}\right)\right]+\left(1-m_{i}\right) \log \left[\frac{1}{2}\left(1-m_{i}\right)\right]\right\} \\
& +\frac{\beta}{2} \sum_{i j} J_{i j} m_{i} m_{j}+\frac{\beta^{2}}{4} \sum_{i j} J_{i j}^{2}\left(1-m_{i}^{2}\right)\left(1-m_{j}^{2}\right)+O\left(\beta^{3}\right) .
\end{aligned}
$$

The first term is the entropy of Ising spins constraint to have magnetization $\left\{m_{i}\right\}$, the second one is minus the 'naïve' mean-field energy, and the third corresponds to the Onsager reaction term. In Ref. [11] the TAP equations for SK were re-derived in this way, the higher order terms being negligible in that case. The analysis of the previous section $\left(m_{i}=0\right)$ tells us that for a model with an orthogonal matrix, the high-temperature series will include an infinite number of non-negligible terms. We must therefore find a systematic way of calculating all the terms in the Gibbs free energy.

In standard field theory, the Legendre transform can be formulated in diagrammatical terms. The Gibbs free energy, also called the effective potential in particle physics, is given by the sum over connected one-particle-irreducible diagrams. This is not true in our case. The reason is that the external field $h_{i}$ does not appear as a linear source term in Eq. (10), instead it appears inside the potential. A shift in $\phi_{i}$ would not simplify matters since it would introduce a term quadratic in $h_{i}$ sufficient to render invalid the usual 1PI derivation. The present authors don't know of any diagrammatic expansion of the Gibbs free energy for Ising-like system.

It is however possible to perform such an expansion algebraically and to give it a diagrammatic interpretation [12]. The weak point of this method is that the vertex weight and the combinatorial factors cannot be calculated systematically. Nevertheless, one can see that the cactus diagrams? will appear in the Gibbs free energy with the same weight but with an extra factor of $\left(1-m_{i_{k}}^{2}\right)$ for each vertex $i_{k}$ [13. Therefore we have

$$
\begin{aligned}
-\beta \Phi= & -\frac{1}{2} \sum\left\{\left(1+m_{i}\right) \log \left[\frac{1}{2}\left(1+m_{i}\right)\right]+\left(1-m_{i}\right) \log \left[\frac{1}{2}\left(1-m_{i}\right)\right]\right\}+\frac{\beta}{2} \sum_{i j} J_{i j} m_{i} m_{j} \\
& +\frac{\beta^{2}}{4} \sum_{i j} J_{i j}^{2}\left(1-m_{i}^{2}\right)\left(1-m_{j}^{2}\right)-\frac{\beta^{4}}{4} \sum_{i j k}\left(1-m_{i}^{2}\right) J_{i j}^{2}\left(1-m_{j}^{2}\right) J_{j k}^{2}\left(1-m_{k}^{2}\right) \\
& +\frac{\beta^{4}}{8} \sum_{i j k l}\left(1-m_{i}^{2}\right) J_{i j}\left(1-m_{j}^{2}\right) J_{j k}\left(1-m_{k}^{2}\right) J_{k l}\left(1-m_{l}^{2}\right) J_{l i}+O\left(\beta^{6}\right) .
\end{aligned}
$$

We now argue that in the large $N$ limit, all those terms (except for the 'entropic' and 'energetic' one) are self-averaging, that is, the error introduced by substituting $m_{k}^{2}$ by $q \equiv N^{-1} \sum_{i} m_{i}^{2}$ vanishes in the thermodynamical limit. Equivalently in sK the Onsager

\footnotetext{
${ }^{2}$ What we call cactus diagrams are called 'loop diagrams' in Ref. [12]. The difference in terminology arises from the fact that these authors consider restricted sums (sums over different indices with combinatorial factors) while we consider unrestricted sums. The vertex weights coming from the expansion of $\log \cosh x$ are precisely those necessary to go from one type of sum to the other.
} 
term in the free energy is often replaced by $\beta^{2}(1-q)^{2} / 4$. We can now resum Eq. (18), by noticing that we are now summing over the same diagrams than in the $m=0$ case. We recover the reaction term of Eq. (13) with $\beta$ replaced by $\beta(1-q)$,

$$
\begin{aligned}
\beta \Phi= & \frac{1}{2} \sum\left\{\left(1+m_{i}\right) \log \left[\frac{1}{2}\left(1+m_{i}\right)\right]+\left(1-m_{i}\right) \log \left[\frac{1}{2}\left(1-m_{i}\right)\right]\right\} \\
& -\frac{\beta}{2} \sum_{i j} J_{i j} m_{i} m_{j}-N G(\beta(1-q))
\end{aligned}
$$

where the function $G(x)$ is again given by Eq. (6).

The same result can be obtained in a more heuristic way. We saw in the previous section that the spherical model reproduces the high-temperature expansion of the model with Ising spins except for the entropic term. It is quite plausible that the two models will have the same Gibbs free energy, except once again for the entropic term. This argument has been used (the other way around) to deduced the right TAP equations for the spherical p-spin model [14]. The general Gibbs free energy for a spherical model with quadratic interaction is given by

$$
\beta \Phi_{\mathrm{sph}}=\log \operatorname{det}^{1 / 2}(\lambda-\beta \mathbf{J})-\frac{N}{2}(\log 2 \pi+\lambda)+\frac{1}{2} \sum_{i j} m_{i}\left(\lambda \delta_{i j}-\beta J_{i j}\right) m_{j},
$$

with $\lambda$ determined by the saddle point equation. Specializing to the case where the eigenvalues of $\mathbf{J}$ are $\{+1,-1\}$ in equal proportions and after eliminating $\lambda$ we find

$$
\beta \Phi_{\mathrm{sph}}=-\frac{N}{2}(\log [2 \pi(1-q)]+1)-\frac{\beta}{2} \sum_{i j} J_{i j} m_{i} m_{j}-N G(\beta(1-q)),
$$

with $q \equiv N^{-1} \sum_{i} m_{i}^{2}$. The first term is the entropy of spherical spins constrained to have magnetization $m_{i}$. As expected, it is the only term that differs from Eq. (19). Note that in this case we did not need to substitute $1-m_{k}^{2}$ with its average value $(1-q)$.

Armed with the free energy (19), we can now write down the 'mean-field' or TAP equations for our model. They are given by the partial derivatives of $\Phi$ with respect to the $m_{i}$ 's:

$$
\tanh ^{-1} m_{i}+2 \beta G^{\prime}(\beta(1-q)) m_{i}-\beta \sum_{j} J_{i j} m_{j}=0
$$

We note finally that if we substitute in Eqs. (19, 22) $G(x)=x^{2} / 4$ we recover the standard result of [15] for sK just as the same substitution in our integration formula (5) recovers the integration over a Gaussian distribution. In Sect. 1 , this fact will provide us with an easy way to compare our formulae against those of Ref. [16].

\section{Minimum of the Free Energy}

The orthogonality of $J$ imposes a simple bound on the energy of any spin configuration. Indeed, a configuration vector $\left\{\sigma_{i}\right\}$ can be decomposed into its projection $\left\{s_{i}^{+}\right\}$on the 
eigenspace with eigenvalue +1 and $\left\{s_{i}^{-}\right\}$on the complement with eigenvalue -1 . Then we have

$$
E(\sigma)=-\frac{1}{2} \sum_{i j} J_{i j} \sigma_{i} \sigma_{j}=-\frac{1}{2}\left(\left|\mathbf{s}^{+}\right|^{2}-\left|\mathbf{s}^{-}\right|^{2}\right) .
$$

In other words, the energy is bounded below by $-N / 2$ and this bound is reached iff $\sigma$ is an eigenvector of $\mathbf{J}$ with eigenvalue +1 . We shall argue later that for a generic large orthogonal matrix the existence of an eigenvector whose every component is either +1 or -1 is highly improbable. Nevertheless, we can construct such a matrix, for example the sine model for odd $N$ with $2 N+1$ a prime number. Whether or not FFM admits such a ground state for special values of $d$ and/or in the limit $d \rightarrow \infty$ is still an open question. Unfortunately, neither an $e=-1 / 2$ spin configuration for $d \geq 9$ has been found nor has a proof that it cannot exist.

For the remaining part of this section, we will consider a model that admits an $e=-1 / 2$ ground state. The TAP equations (22) admit in this case a solution of the form

$$
m_{i}=\sqrt{q} \epsilon_{i}
$$

where the $\left\{\epsilon_{i}\right\}$ are \pm 1 and form an eigenvector of $\mathbf{J}$ with eigenvalue +1 . With this ansatz Eq. (22) reduces to

$$
q=\tanh ^{2}\left\{\beta \sqrt{q}\left[1+\frac{1-\sqrt{1+4 \beta^{2}(1-q)^{2}}}{2 \beta(1-q)}\right]\right\}
$$

where we have used Eq. (6) to compute $G^{\prime}(x)$. This solution has specific free energy given by

$$
\beta f=\frac{1+\sqrt{q}}{2} \log \left[\frac{1}{2}(1+\sqrt{q})\right]+\frac{1-\sqrt{q}}{2} \log \left[\frac{1}{2}(1-\sqrt{q})\right]-\frac{\beta}{2} q-G(\beta(1-q)) .
$$

For a given value of $q$, this is the lowest free energy solution. In fact, the energetic term reaches its lower bound from orthogonality, the entropy is maximum (at fixed $q$ ) when all the local magnetizations are equal in magnitude and the reaction term only depends on $q$. The absolute minimum of the free energy must therefore be of this form. Eq. (25) always admits a solution with $q=0$. At low temperature it will also admit a solution with non-zero $q$. Numerically one finds that below $T=0.400^{3}$ a solution with $q=0.92$ appears but with higher free energy that the paramagnetic solution. At $T=0.178(q=0.99995)$ this solution becomes the true minimum of the free energy.

One might conclude as in [13], that the system undergoes a first order transition at $T=0.178$. This transition is not seen in Monte Carlo simulations of FFM or the sine model when cooling down from high temperature. Besides Eq. (24), there are many other solutions to the mean-field equations. As we shall we in the next section, for a

\footnotetext{
${ }^{3}$ This paper contains many numbers obtained from numerical analysis, they are noted with equal signs and every digit is significant.
} 
generic interaction matrix and below a certain critical temperature, their number grows exponentially with the size of the system. It is those solutions and the large free energy barriers between them that prevent the system from finding the true minimum of the free energy and gives it instead a glassy behavior.

When the ground state configuration is known - as in the sine model for special $N$ it is however possible to see this 'crystalline state' in Monte Carlo simulations. One has to start in the ground state at $T=0$ and continuously increase the temperature. Fig. 10 of Ref. [6] shows the result of such a simulation (sine model $N=44$ and $N=806$ ). Calculation of the internal energy for our solution reproduces exactly those curves. Note that for the larger value of $N$ the system stays trapped beyond the point where the paramagnetic solution becomes stable $(T=0.712$ on their scale) this is a clear sign that barriers between different metastable states are very high.

The solution (24) can be transposed directly to the FFM on the lattice by taking care of the appropriate factors of 2 and $\sqrt{2}$ recovering the result of Ref. [13. One important point, though, is that this solution is only valid if the ground state energy density is $-1 / 2$ ( -1 for the full lattice), otherwise $\left\{\epsilon_{i}\right\}$ is not an eigenvector of $\mathbf{J}$ and Eq. (22) does not reduce to Eq. (25).

\section{Number of TAP Solutions}

\subsection{General Result}

In this section we will compute the average number of metastable states for ROM. We will follow as much as possible the steps and the notation of Ref. [16] where the corresponding result for SK was first derive. Recall our TAP equations:

$$
\begin{aligned}
\Gamma_{i} & \equiv \tanh ^{-1} m_{i}+2 \beta G^{\prime}(\beta(1-q)) m_{i}-\beta \sum_{i \neq j} J_{i j} m_{j}=0 \\
& \equiv g\left(m_{i}\right)-\beta \sum_{i \neq j} J_{i j} m_{j}=0,
\end{aligned}
$$

with corresponding free energy written as a sum of single site terms:

$f=(\beta N)^{-1} \sum_{i}\left[-\log 2-G(\beta(1-q))-\beta q G^{\prime}(\beta(1-q))+\frac{1}{2} m_{i} \tanh ^{-1} m_{i}+\frac{1}{2} \log \left(1-m_{i}^{2}\right)\right]$.

We write the number of solutions as the integral over all possible values of $m_{i}$ fixing with $\delta$-functions that the $\left\{m_{i}\right\}$ form a solution of Eq. (27) with free energy $f$. Using a Fourier representation of the $\delta$-function, we obtain

$$
\begin{aligned}
\mathcal{N}_{s}(f)= & N^{2} \int_{0}^{1} d q \int_{-\mathrm{i} \infty}^{\mathrm{i} \infty} \frac{d \lambda}{2 \pi \mathrm{i}} \int_{-\mathrm{i} \infty}^{\mathrm{i} \infty} \frac{d u}{2 \pi \mathrm{i}} \int_{-\mathrm{i} \infty}^{\mathrm{i} \infty} \prod_{i}\left(\frac{d x_{i}}{2 \pi \mathrm{i}}\right) \int_{-1}^{1} \prod_{i}\left(d m_{i}\right) \exp [-N(\lambda q+u f) \\
& \left.-\lambda \sum_{i} m_{i}^{2}+u \sum_{i} f\left(m_{i}\right)+\sum_{i} x_{i} g\left(m_{i}\right)-\beta \sum_{i<j} J_{i j}\left(x_{i} m_{j}+x_{j} m_{i}\right)\right]|\operatorname{det} \mathbf{A}|(29)
\end{aligned}
$$


where

$$
\begin{aligned}
A_{i j} & =\frac{\partial \Gamma_{i}}{\partial m_{j}}=\left[\left(1-m_{i}^{2}\right)^{-1}+2 \beta G^{\prime}(\beta(1-q))\right] \delta_{i j}-\beta J_{i j} \\
& \equiv a_{i} \delta_{i j}-\beta J_{i j}
\end{aligned}
$$

dropping a term in $m_{i} m_{j} / N$ (see appendix).

We can now proceed to average Eq. (29) over the random couplings $J_{i j}$. We should really be averaging $\log \mathcal{N}_{s}$, the extensive quantity, not $\mathcal{N}_{s}$. To do so we would need to introduced replicas. The replica symmetric computation would be tedious but straightforward leaving us not with 7 unknown parameters (as will be the case below) but on the order of 14 . On the other hand, the direct average will be sufficient to provide us a clear picture of the metastable states.

There are two terms that depend on the $\left\{J_{i j}\right\}$. It is shown in appendix that the two can be averaged independently. Using Eq. (5) to average the term involving $x_{i}$ and $m_{i}$, we find

$$
\begin{aligned}
\left\langle\exp \left[-\beta \sum_{i<j} J_{i j}\left(x_{i} m_{j}+x_{j} m_{i}\right)\right]\right\rangle & =\exp \left\{N \operatorname{Tr} G\left[\beta \frac{\mathbf{x} \otimes \mathbf{m}+\mathbf{m} \otimes \mathbf{x}}{N}\right]\right\} \\
& =\exp \{N[G(v+\sqrt{w})+G(v-\sqrt{w})]\}
\end{aligned}
$$

where

$$
v=\frac{\beta}{N} \sum_{i} x_{i} m_{i} \text { and } w=\frac{\beta^{2} q}{N} \sum_{i} x_{i}^{2} .
$$

The second equality in Eq. (31) follows from an analysis of the eigenvalues of the matrix $\mathbf{x} \otimes \mathbf{m}+\mathbf{m} \otimes \mathbf{x}$. This matrix has only two non-zero eigenvalues corresponding to the two terms: $v+\sqrt{w}$ and $v-\sqrt{w}$.

To compute the average the determinant we will need to drop the absolute value. This corresponds to weighting each solution with the sign of the determinant of its Hessian matrix. Formally, we would be computing a topological invariant (from Morse theory) which as little to do with our original goal. Nevertheless, the calculation without the absolute value gives sensible results (here and in Refs. [16, 17]) and connects smoothly with the zero temperature results where the calculation can be done without this pathology. This problem is discussed in more detail in Refs. [18, 19].

We introduce a set of anti-commuting (Grassman) variables $\left\{\theta_{i}, \theta_{i}\right\}$ to express the determinant as an exponential,

$$
\begin{aligned}
\langle\operatorname{det} \mathbf{A}\rangle & =\left\langle\int d \boldsymbol{\theta} d \overline{\boldsymbol{\theta}} \exp \left[\sum_{i} a_{i} \bar{\theta}_{i} \theta_{i}-\beta \sum_{(i j)} J_{i j}\left(\bar{\theta}_{i} \theta_{j}+\bar{\theta}_{j} \theta_{i}\right)\right]\right\rangle \\
& =\int d \boldsymbol{\theta} d \overline{\boldsymbol{\theta}} \exp \left\{\sum_{i} a_{i} \bar{\theta}_{i} \theta_{i}+N \operatorname{Tr} G\left[\beta \frac{\overline{\boldsymbol{\theta}} \otimes \boldsymbol{\theta}-\boldsymbol{\theta} \otimes \overline{\boldsymbol{\theta}}}{N}\right]\right\} .
\end{aligned}
$$


If we expand $\operatorname{Tr} G[\ldots]$ in a Taylor series, we can use the following propriety of Grassman variables

$$
\left(\bar{\theta}_{i_{1}} \theta_{i_{2}}+\bar{\theta}_{i_{2}} \theta_{i_{1}}\right)\left(\bar{\theta}_{i_{2}} \theta_{i_{3}}+\bar{\theta}_{i_{3}} \theta_{i_{2}}\right) \ldots\left(\bar{\theta}_{i_{n}} \theta_{i_{1}}+\bar{\theta}_{i_{1}} \theta_{i_{n}}\right)=-2 \prod_{k=1}^{n} \bar{\theta}_{i_{k}} \theta_{i_{k}}
$$

which allows us to resum the series and find

$$
\langle\operatorname{det} \mathbf{A}\rangle=\int d \boldsymbol{\theta} d \overline{\boldsymbol{\theta}} \exp \left\{\sum_{i} a_{i} \bar{\theta}_{i} \theta_{i}-2 N G\left[\frac{\beta}{N} \sum_{i} \bar{\theta}_{i} \theta_{i}\right]\right\} .
$$

Finally we fix $r=(\beta / N) \sum_{i} \bar{\theta}_{i} \theta_{i}$ using a Lagrange multiplier $R$, which allows us to perform the integration over the Grassman variables, leaving us with

$$
\langle\operatorname{det} \mathbf{A}\rangle=\int_{-\infty}^{\infty} d r \int_{-\mathrm{i} \infty}^{\mathrm{i} \infty} \frac{d R}{2 \pi \mathrm{i}} \prod_{i}\left(a_{i}+\beta R\right) \exp \{N[-r R-2 G(r)]\} .
$$

We now collect results form Eqs. (29), (31) and (36), introduce Lagrange multipliers $V$ and $W$ to impose Eqs. (32), perform the $x_{i}$ integration, drop multiplicative prefactors and finally set $V=2 G^{\prime}(\beta(1-q))-\Delta, R=B / \beta-2 G^{\prime}(\beta(1-q))$ and $r=\beta b$, to obtain

$$
\begin{aligned}
\left\langle\mathcal{N}_{s}(f)\right\rangle= & \text { saddle } \exp \{N[-\lambda q-u f-b B-v \Delta-w W / q+G(v+\sqrt{w}) \\
& \left.\left.+G(v-\sqrt{w})-2 G(\beta b)+2(\beta b-v) G^{\prime}(\beta(1-q))+\log I\right]\right\}
\end{aligned}
$$

where

$$
I=\int_{-1}^{1} \frac{d m}{2 \beta \sqrt{\pi W}}\left(\frac{1}{1-m^{2}}+B\right) \exp \left[-\frac{\left(\tanh ^{-1} m-\beta \Delta m\right)^{2}}{4 \beta^{2} W}+\lambda m^{2}+u f(m)\right]
$$

We have indicated by the term saddle that the right-hand side of Eq. (37) is to be extremized with respect to the nine variables: $\lambda, q, u, b, B, v, \Delta, w, W$. By partial differentiation with respect to those variables, we obtain the saddle point equations. They admit a solution with $B=0$ which implies $b=1-q$; following Ref. [16] we adopt this solution. This choice will also lead us to the correct $T=0$ result.

As advertised earlier, the result for SK can be obtained from Eq. (37) by setting $G(x)=x^{2} / 4$. In this case, three variables can be eliminated using the saddle point equations to give back the same expression as in Ref. [16].

\section{2 $T=0$ Result}

Before looking at the numerical solution for the saddle point of Eq. (37), let us study its $T=0$ limit. In this limit, $q \rightarrow 1, \lambda$ decouples, and the five other parameters have 
a finite value. The integral in Eq. (38) can then be done analytically using a change of variable $m=1-\exp (-\beta y)$ and keeping only terms with a finite limit when $\beta \rightarrow \infty$,

$$
\lim _{\beta \rightarrow \infty} I=\exp \left(u^{2} W / 4-\Delta u / 2\right) \operatorname{erfc}\left(\frac{u W-\Delta}{2 \sqrt{W}}\right) .
$$

Shifting $\Delta$ and doing the integral over $u$, we obtain

$$
\begin{aligned}
\left\langle\mathcal{N}_{s}(f)\right\rangle= & \text { saddle } \exp \left\{N \left[-v \Delta-w W+(f+v W+\Delta / 2)^{2} / W+G(v+\sqrt{w})\right.\right. \\
& +G(v-\sqrt{w})+\log \operatorname{erfc}(-\Delta / 2 \sqrt{W})]\} .
\end{aligned}
$$

The same result can be obtained directly by counting the average number of spin configurations $\left\{\sigma_{i}\right\}$ satisfying

$$
\forall i \sigma_{i} \sum_{j \neq i} J_{i j} \sigma_{j}>0 \text { and } f=-\sum_{i<j} J_{i j} \sigma_{i} \sigma_{j}
$$

This computation can be done in rather straightforward way using Heaviside step functions instead of $\delta$-functions. This approach is free of the problem of the absolute value of the determinant.

The saddle point equations obtained from Eq. (40) were solved numerically to give the shape of the distribution of one-flip stable configurations as a function of energy density (Fig. 2 full-line). Note that since Eq. (40) involves the function $G(x)$ for potentially complex arguments (the complex conjugate term assures us that the final result will always be real) one has to choose a sign convention for the square-root terms in the definition of $G(x)$ (c.f. Eq. (6)) ). It was chosen such that the real part of the square-root is always of the same sign as $v$ in $G(v+\sqrt{w})$; this way the saddle point never has to cross any cut in the complex plane.

As one follows the saddle point to the rightmost part of the curve $(e>-0.36)$, the argument of the error-function in Eq. (40) goes through infinity after which all four parameters become complex. It is not clear if the contour of the integral computed by the saddle point method can be deformed to have this complex saddle point has its main contribution. This issue was not investigated any further for it is of marginal interest.

In addition, an approximate enumeration of the one-flip stable configurations for a relatively small $(N=48)$ ROM was done. Random configurations were generated and then cooled at zero temperature, the resulting one-flip stable configurations were compared with previously stored one and were stored if not previously obtained. After $4 \times 10^{6}$ iterations, the low-energy configurations were each found about a hundred times while only a few high-energy ones remained unfound. The resulting distribution was binned and its logarithm (divided by 48) is shown as the data points on Fig. 2. An overall normalization constant (not predicted by the saddle point method) was added to the simulation data to make them fall on the predicted curve. Points on the xaxis correspond to energy bins where no metastable states were found. The agreement 
between the theory and this simulation is very good. Similar data (not shown) for the sine-model also gave very good agreement with the ROM prediction. For FFM the Diophantine constraints (integer spin and integer field) are such that for small $d$ only a few values of the energy are allowed for a metastable state (e.g. 2 for $d=5$ and 3 for $d=6)$. Those constraints might not be so important for large $d$, but for $d \geq 6(N \geq 64)$ an exhaustive search becomes impossible. Whether the distribution of metastable states in FFM follows that of ROM remains an open question.

The point where the curve shown in Fig. 3 intersects the x-axis on the left, corresponds to the minimum energy $\left(E_{\mathrm{NSol}}\right)$ at which there are on average exponentially many configurations. It is almost equal to the approximate ground-state energy $\left(E_{1 \text {-step }}\right)$ from the one-step replica breaking solution of this model [6]. Careful analysis of the equations leading to these two quantity done using arbitrary precision arithmetics shows that while they are not equal they differ by less than 30 parts in a billion. Precisely, $E_{\mathrm{NSol}}=-0.484119415$ and $E_{1 \text {-step }}=-0.484119428$. The true average ground state energy density for ROM is probably well approximated by either of these two quantities.

\subsection{Finite $T$ Results}

By taking partial derivatives of Eq. (37), we can write the number of metastable states in terms of the solution of six coupled non-linear equations, three of which containing definite integrals. A numerical solution of these equations was done by following the $T=0$ solution of the previous section to finite $T$. At fixed $T$, varying $u$ traces out the $N^{-1} \log \left\langle\mathcal{N}_{s}\right\rangle$ vs $f$ curve. Typical curves (for $T=0.134$ and $T=0.065$ ) are shown on Fig. 2 (dash line and dotted line); results for other temperature are quite similar. The abrupt stop on the right-hand side of these curves is due to the divergence of the definite integral in Eq. (37), it is possible that the saddle point can be analytically continued to complex values giving a smooth curve all the way to zero as in the $T=0$ case. This issue has not been investigated since the interesting points lie on the low free energy end of the curve.

Setting $u=0$ in Eq. (37) gives the total number of TAP solutions. It gives a positive value for $N^{-1} \log \left\langle\mathcal{N}_{s}\right\rangle$ starting from 0.2854 at $T=0$ and decreasing all the way to 0 at $T=0.32$. Above this temperature, the $q=0$ saddle point with $\log \left\langle\mathcal{N}_{s}\right\rangle=0$ is the correct one. The full results are plotted in Fig. 3 (full line).

In the thermodynamics of the model nothing special happens at the temperature $T=0.32$ at which exponentially many TAP solutions appear. These metastable states have too high free energy to contribute to the partition function. Instead, if we write

$$
e^{-\beta F}=\sum_{\alpha} e^{-\beta F_{\alpha}} \approx \int d f\left\langle\mathcal{N}_{s}(f)\right\rangle e^{-N \beta f} \approx \max _{f} e^{N A(f)}
$$

where $\alpha$ labels TAP solutions and with

$$
A(f) \equiv N^{-1} \log \left\langle\mathcal{N}_{s}(f)\right\rangle-\beta f
$$


we find that the solutions which contributes to the free energy are those for which $A(f)$ is maximum. They are given by setting $u=-\beta$ in Eq. (37). With this substitution, the saddle point equations admit a simple solution of the form:

$$
v=\beta-\frac{\beta q}{2}, w=\frac{\beta^{2} q^{2}}{4}, \Delta=G^{\prime}(\beta)-G^{\prime}(\beta(1-q)), W=\frac{\Delta}{\beta} \text { and } \lambda=\frac{\beta \Delta}{4} .
$$

And the number of contributing TAP solutions reduces to

$$
\left\langle\mathcal{N}_{c s}\right\rangle=\exp \{N(\beta f+G(\beta)+\log 2)\}
$$

where

$$
\begin{aligned}
f=\beta^{-1}[ & -\log 2-G(\beta(1-q))-\beta q G^{\prime}(\beta(1-q))+\beta \Delta \\
& \left.-e^{-\beta \Delta} \int_{-\infty}^{\infty} \frac{d z}{\sqrt{2 \pi}} e^{-\frac{z^{2}}{2}} \cosh (\sqrt{2 \beta \Delta} z) \log \cosh (\sqrt{2 \beta \Delta} z)\right]
\end{aligned}
$$

with $\Delta(q)$ as above and $q$ determined variationally. The function $f$ corresponds the free energy of the contributing solutions. We notice that the logarithm of the number of contributing solutions, often called complexity, can be written as

$$
\log \left\langle\mathcal{N}_{c s}\right\rangle=\left.\frac{\partial}{\partial m} \beta F_{1-\text { step }}(\beta, m, q)\right|_{m=1}
$$

where $F_{1 \text {-step }}$ is the free energy computed with one-step replica symmetry breaking with RSB parameter $m, q_{0}=0$ and extremized over $q_{1}=q$ (Eq. (44) in Ref. [6]). Equality (47) is in fact more general than what is presented in here, for a more direct derivation and its physical interpretation see Ref. [20].

The condition for Eq. (45) to have a saddle point at a non-zero value of $q$ is equivalent to the marginality condition $\left(T<T_{\mathrm{M}}=0.134\right)$. At this temperature, one finds $q=0.962$ with $N^{-1} \log \left\langle\mathcal{N}_{c s}\right\rangle=0.158$. Monte Carlo simulations have indicated that all three orthogonal models considered here (FFM, ROM and the sine model) undergo a dynamical glassy transition at a temperature equal or very near $T_{\mathrm{M}}$ [6, 10].

The complexity goes to zero at the replica symmetry breaking transition $T_{\mathrm{RSB}}=$ 0.065. For temperatures below $T_{\mathrm{RSB}}$, Eq. (45) gives an unphysical negative complexity: the above saddle point is no longer valid and the contributing solutions are those with the smallest free energy (the leftmost points of the curves of Fig. 2). The full curve of complexity vs. temperature is plotted on Fig. 3 (dash line).

We finally notice that we naturally have

$$
\max _{f} A(f)=\log 2+G(\beta)=-\beta f_{\mathrm{RS}} .
$$

In other words even in the region between $T_{\mathrm{RSB}}$ and $T_{\mathrm{M}}$, where a large number of solutions contribute to the free energy, the Rs free energy is still valid. The decomposition of the 


\begin{tabular}{|l|l|}
\hline Temp. & Comments \\
\hline 0.40 & Metastable crystalline phase (if present) \\
0.32 & Exponential number of TAP solutions \\
0.18 & Stability of crystalline solution (if present) \\
0.134 & TAP solutions start contributing to the free energy, 'marginality condition' \\
& $\begin{array}{l}\text { and dynamical phase transition } \\
0.065\end{array}$ Complexity goes to zero and replica symmetry breaking \\
\hline
\end{tabular}

Table 1: Interesting temperatures for the orthogonal model, from text and adapted from Ref. [6] to present normalizations.

RS free energy into contributions from many metastable states below a temperature " $T_{\mathrm{g}}$ " higher than $T_{\mathrm{RSB}}$ has been discussed in the context of many different models [21, 22, 23, 24 .

Above $T_{\mathrm{M}}$, one can still find a saddle point of Eq. (37) with $u=-\beta$, corresponding to the maximum of $A(f)$, but it is no longer of the simple form (44). Numerically one finds that for those temperature, $\max A(f)<-\beta f_{\mathrm{RS}}$. The metastable states don't contribute to the free energy.

It may seem surprising that the RS solution is still valid while a very large number of different states contribute to the free energy. The explanation is that their number is so large that if ones chooses randomly two such states the probability of finding the same one is zero, therefore the order parameter $P(q)$ only measures the overlap between different states which is always equal to $q_{0}=0$. Only when the complexity ceases to be extensive can the $P(q)$ become non-trivial, leading to replica symmetry breaking.

We now have a simple intuitive scenario for the phase diagram of models such as ROM where the order parameter jumps discontinuously at the transition (see Table 1). At a relatively high temperature $(T=0.32$ in ROM $)$ the number of metastable states increases dramatically, but their free energy is so high that they influence neither the static nor the dynamics. At $T_{\mathrm{M}}$, a large number of these states start contributing to the free energy. At this temperature, all static thermodynamical quantities are perfectly regular, but the time scales involved with the dynamics diverge and the system is no longer able to thermalize. Phenomena such as aging start to appear. At $T_{\mathrm{RSB}}$ the entropy of the contributing states goes to zero, $P(q)$ becomes non-trivial and replica symmetry is broken.

\section{Two-Group model}

In this final section we will divert a bit our analysis of the thermodynamics of the orthogonal model to look at a puzzling analytical 'coincidence.' Indeed, by computing the partition function for a certain replica-symmetry breaking scheme, we will recover the expression for the average number of TAP solution. The 'two-group model' was first 
introduced in Ref. [25] as an attempt to break replica symmetry in SK. It was later noticed [26] that the partition function $\left(\lim _{n \rightarrow 0}\left\langle Z_{2 \mathrm{G}}^{n}\right\rangle_{J}\right)$ in this framework is not equal to unity but instead one has

$$
\left\langle Z_{2 \mathrm{G}}^{n}\right\rangle=\int d f\left\langle\mathcal{N}_{s}(f)\right\rangle e^{-n \beta N f},
$$

and in particular $\lim _{n \rightarrow 0}\left\langle Z_{2 \mathrm{G}}^{n}\right\rangle_{J}$ gives the average number of TAP solutions $\left(\left\langle\mathcal{N}_{s}\right\rangle_{J}\right)$. We present here a general derivation of the partition function for the 'two-group model.' We will assume that the coupling matrix is chosen from a distribution that obeys a relation such as Eq. (5) but we will not make use of the explicit form of the function $G(x)$. We will recover the result for SK as the special case $G(x)=x^{2} / 4$.

The average partition function for ROM with $n$ replica is given by

$$
\left\langle Z^{n}\right\rangle_{J}=\int d \mathbf{Q} d \boldsymbol{\Lambda} \exp \left\{N\left[\operatorname{Tr} G(\beta \mathbf{Q})-\frac{1}{2} \operatorname{Tr}(\boldsymbol{\Lambda} \mathbf{Q})+\log Z_{\circ}(\boldsymbol{\Lambda})\right]\right\},
$$

with

$$
Z_{\circ}(\boldsymbol{\Lambda}) \equiv \sum_{\sigma^{a}} \exp \left\{\sum_{a<b} \Lambda_{a b} \sigma^{a} \sigma^{b}\right\} .
$$

The two-group ansatz correspond to writing the matrix $\boldsymbol{\Lambda}$ and $\mathbf{Q}$ as made up of two diagonal blocks of size $m \times m$ and $(n-m) \times(n-m)$ and equal elements outside those blocks. We specialize right away to the case $n=0$ which will make some of our formulae look a bit strange but will make the notation more compact. The following derivation can be extended without much difficulties to the $n \neq 0$ case. We define

$$
\boldsymbol{\Lambda}=(\overbrace{u_{1}}^{m} \overbrace{u}^{-m}) \text { and } \mathbf{Q}=\left(\begin{array}{ccc}
\overbrace{q_{1}} & \overbrace{q}^{m} \\
q & q_{2}
\end{array}\right),
$$

with $\Lambda_{a a}=0$ and $Q_{a a}=1$ and with the elements parameterized as

$$
\begin{gathered}
u_{1}=u+t / m+r / m^{2}, u_{2}=u-t / m+r / m^{2} \\
q_{1}=q+\alpha / m+\gamma / m^{2}, q_{2}=q-\alpha / m+\gamma / m^{2},
\end{gathered}
$$

where $m$ will be taken to infinity. Having defined the two-group ansatz, we can now compute the different parts of Eq. (50);

$$
\operatorname{Tr}(\boldsymbol{\Lambda} \mathbf{Q})=2(\alpha t+q r+\gamma u)-2(\alpha u+q t) .
$$

To compute $\operatorname{Tr} G(\beta \mathbf{Q})$ we find the different eigenvalues of the matrix $\mathbf{Q}$ and their multiplicity, leading us to

$$
\begin{aligned}
\operatorname{Tr} G(\beta \mathbf{Q}) & =\lim _{m \rightarrow \infty}(m-1) G\left(\beta\left(1-q_{1}\right)\right)-(m+1) G\left(\beta\left(1-q_{2}\right)\right)+G\left(\beta q^{+}\right)+G\left(\beta q^{-}\right) \\
& =-2 G(\beta(1-q))-2 \alpha \beta G^{\prime}(\beta(1-q))+G\left(\beta q^{+}\right)+G\left(\beta q^{-}\right),
\end{aligned}
$$


where

$$
q^{ \pm}=q-\alpha-1 \pm \sqrt{2 q(\gamma-\alpha)}
$$

The $Z_{\circ}(\boldsymbol{\Lambda})$ term requires more work,

$$
Z_{\circ}(\boldsymbol{\Lambda})=\lim _{m \rightarrow \infty} e^{-t} \sum_{\sigma^{a}} \exp \left\{\frac{1}{2}\left[u S^{2}+\left(\frac{t}{m}+\frac{r}{m^{2}}\right) S_{+}^{2}+\left(-\frac{t}{m}+\frac{r}{m^{2}}\right) S_{-}^{2}\right]\right\},
$$

where

$$
S=\sum \sigma_{a}, S_{+}=\sum_{a \leq m} \sigma_{a} \text { and } S_{-}=\sum_{a>m} \sigma_{a}
$$

If we make three Hubbard-Stratonovich transformations to linearize the quadratic terms and do the sum over spins, we find

$$
\begin{aligned}
Z_{\circ}(\boldsymbol{\Lambda})= & \lim _{m \rightarrow \infty} e^{-t} \int_{-\infty}^{\infty} \frac{d h}{\sqrt{2 \pi u}} \int_{-\infty}^{\infty} \int_{-\infty}^{\infty} \frac{m d h_{+} d h_{-}}{2 \pi t} \exp \left\{\frac{1}{2}\left[-\frac{h^{2}}{u}+\frac{r\left(h_{+}^{2}+h_{-}^{2}\right)}{t^{2}}\right]\right. \\
& \left.+m\left[\log \cosh \left(h+h_{+}\right)-\log \cosh \left(h+h_{-}\right)-\frac{h_{+}^{2}}{2 t}+\frac{h_{-}^{2}}{2 t}\right]\right\},
\end{aligned}
$$

where we have put in evidence the terms with a factor of $m$. We can now use the saddle point method to compute the $m \rightarrow \infty$ limit of the last expression. To leading order in $m$, the saddle point equations for $h_{+}$and $h_{-}$are the same. They do differ by a term of order $1 / m$ but this perturbation only contributes to the saddle point at order $1 / m$ (it does not contribute at order one because the derivative of the order $m$ term vanishes). At the saddle point, one has $h_{+}=h_{-}=h_{\circ}$ which solves

$$
E\left(h, h_{\circ}\right) \equiv \tanh \left(h+h_{\circ}\right)-\frac{h_{\circ}}{t}=0 .
$$

The order $m$ contributions to the saddle point of $h_{+}$and $h_{-}$exactly cancel each other leaving us with a finite result as $m \rightarrow \infty$. Including Gaussian fluctuations, we find

$$
Z_{\circ}(\boldsymbol{\Lambda})=e^{-t} \int_{-\infty}^{\infty} \frac{d h}{t \sqrt{2 \pi u}}\left(\frac{\partial E}{\partial h_{\circ}}\right)^{-1} \exp \left[\frac{1}{2}\left(-\frac{h^{2}}{u}+\frac{2 r h_{\circ}^{2}}{t^{2}}\right)\right] .
$$

Using the implicit function theorem,

$$
d h\left(\frac{\partial E}{\partial h_{\circ}}\right)^{-1}=d h_{\circ}\left(\frac{\partial E}{\partial h}\right)^{-1}=d h_{\circ}\left(1-\frac{h_{\circ}^{2}}{t^{2}}\right)^{-1},
$$

we can change the integration variable from $h$ to $h_{\circ}$ which we rescalef to find

$$
Z_{\circ}(\boldsymbol{\Lambda})=e^{-t} \int_{-1}^{1} \frac{d m}{\sqrt{2 \pi u}}\left(\frac{1}{1-m^{2}}\right) \exp \left[-\frac{\left(\tanh ^{-1} m-t m\right)^{2}}{2 u}+r m^{2}\right] .
$$

\footnotetext{
${ }^{4}$ Note that we call $m$ the rescaled integration variable $h_{\circ} / t$ to make the connection with Eq. (37), it is not to be confused with the replica-symmetry breaking parameter $m$ which as been taken to infinity.
} 
Finally if we collect the results from Eqs. (55, 56, 64), make the following change of variables

$$
\Delta=t / \beta, W=u / 2 \beta^{2}, \lambda=r, w=\beta^{2}[2 q(\gamma-\alpha)] \text { and } v=\beta(\alpha+1-q),
$$

and extremize over the six parameters, we recover $\left\langle\mathcal{N}_{s}\right\rangle$ given by Eq. (37) with $u=0$ where the saddle point with $B=0$ and $b=1-q$ has been chosen. Had we not set $n=0$, we would have recovered the r.h.s. of Eq. (37) with $u=-n \beta$ and without the $-u f$ term. In other words $\left\langle Z_{2 \mathrm{G}}^{n}\right\rangle$ is the Laplace transform of $\left\langle\mathcal{N}_{s}(f)\right\rangle$ with $-n \beta N$ conjugate to $f$.

\section{Acknowledgments}

We thank J. Kurchan, E. Marinari, R. Monasson, F. Ritort and M. Wexler for useful discussions.

\section{Appendix}

We will now proceed to show that the determinant of the matrix $A$ and the term $\sim J_{i j} x_{i} m_{j}$ (c.f. Eq. (29)) can be averaged separately. Had we kept them together, introduced Grassman variables and integrate over the couplings, we would have obtained a term like this

$$
\exp \left\{N \operatorname{Tr} G\left[\beta \frac{\mathbf{x} \otimes \mathbf{m}+\mathbf{m} \otimes \mathbf{x}+\overline{\boldsymbol{\theta}} \otimes \boldsymbol{\theta}-\boldsymbol{\theta} \otimes \overline{\boldsymbol{\theta}}}{N}\right]\right\}
$$

Therefore we need to show that the cross-terms in the Taylor expansion of $\operatorname{Tr} G[\ldots]$ are irrelevant in the large $N$ limit. A typical cross-term is

$$
\frac{1}{N^{n-1}} \sum_{\left\{i_{k}\right\}} x_{i_{1}} m_{i_{2}} x_{i_{2}} m_{i_{3}} \theta_{i_{3}} \bar{\theta}_{i_{4}} \theta_{i_{4}} \bar{\theta}_{i_{5}} m_{i_{5}} x_{i_{6}} \ldots \bar{\theta}_{i_{n}} \theta_{i_{1}}
$$

We can consider those terms as a perturbation about the fermionic Gaussian integral (35). We need to compute all connected diagrams generated by these new $\theta$ and $\bar{\theta}$ vertices, contracting them with the Gaussian propagator $\left(a_{i}+\beta R\right)^{-1} \delta_{i j}$. The point is that since the propagator is diagonal, all such contractions will be at most of order 1 while the logarithm of the unperturbed result (Eq. (36)) is of order $N$. The same is true of the term we dropped in writing Eq. (30).

\section{References}

[1] J.-P. Bouchaud and M. Mézard, Self Induced Quenched Disorder: A Model for the Glass Transition, J. Phys. I France 4 (1994) 1109. 
[2] E. Marinari, G. Parisi and F. Ritort, Replica Field Theory for Deterministic Models: Binary Sequences with Low Autocorrelation, J. Phys. A (Math. Gen.) 27 (1994) 7615.

[3] G. Parisi, Some Applications of Field Theory to Amorphous Systems, in Quantum Field Theory and Quantum Statistics. Essays in honour of the sixtieth birthday of E. S. Fradkin, edited by I. A. Batalin, C. J. Isham and G. A. Vilkovisky, Adam Hilger (Bristol) 1987.

[4] G.Parisi, Field Theory, Disorder and Simulations, World Scientific (Singapore) 1992.

[5] G. Parisi and V. Dotskenko, Random Magnetic Fields and Instantons in Replica Space, J. Phys. A (Math. Gen.) 25 (1992) 3143.

[6] E. Marinari, G. Parisi and F. Ritort, Replica Field Theory for Deterministic Models (II): A Non-Random Spin Glass with Glassy Behavior, J. Phys. A (Math. Gen.) 27 (1994) 7647.

[7] G. Toulouse, Theory of Frustration Effect in Spin Glasses: I, Comm. Phys. 2 (1977) 115.

[8] B. Derrida, Y. Pomeau, G. Toulouse and J. Vannimenus, Fully Frustrated Simple Cubic Lattices and the Overblocking Effect, J. Physique 40 (1979) 617.

[9] B. Derrida, Y. Pomeau, G. Toulouse and J. Vannimenus, Fully Frustrated Simple Cubic Lattices and Phase Transitions, J. Physique 41 (1980) 213.

[10] E. Marinari, G. Parisi and F. Ritort, The Fully Frustrated Hypercubic Model is Glassy and Aging at Large D, J. Phys. A (Math. Gen.) 28 (1995) 327.

[11] T. Plefka, Convergence Condition of the TAP Equation for the Infinite-Range Ising Spin Glass Model, J. Phys. A (Math. Gen.) 15 (1982) 1971.

[12] A. Georges and J. S. Yedidia, How to Expand around Mean-Field Theory Using High-Temperature Expansions, J. Phys. A (Math. Gen.) 24 (1991) 2173.

[13] J. S. Yedidia and A. Georges, The Fully Frustrated Ising Model in Infinite Dimensions, J. Phys. A (Math. Gen.) 23 (1990) 2165.

[14] J. Kurchan, G. Parisi and M. A. Virasoro, Barriers and Metastable States as Saddle Points in the Replica Approach, J. Phys. I France 3 (1993) 1819

[15] D. J. Thouless, P. W. Anderson and R. G. Palmer, Solution of 'Solvable Model of a Spin Glass', Phil. Mag. 35 (1977) 593. 
[16] A. J. Bray and M. A. Moore, Metastable States in Spin Glasses, J. Phys. C (Solid St. Phys.) 13 (1980) L469.

[17] H. Rieger, The Number of Solutions of the Thouless-Anderson-Palmer Equations for the p-Spin-Interaction Spin Glasses, Phys. Rev. B 46 (1992) 14655.

[18] G. Parisi and N. Sourlas, Supersymmetric Field Theories and Stochastic Differential Equations, Nucl. Phys. B 206 (1982) 321.

[19] J. Kurchan, Replica Trick to Calculate Means of Absolute Values: Applications to Stochastic Equations, J. Phys. A (Math. Gen.) 24 (1991) 4969.

[20] R. Monasson, in preparation.

[21] T. R. Kirkpatrick and D. Thirumalai, $p$-spin Interaction Spin-Glass Models: Connections with the Structural Glass Problem, Phys. Rev. B. 36 (1987) 5388.

[22] T. R. Kirkpatrick and P. G. Wolynes, Stable and Metastable States in Mean-Field Potts and Structural Glasses, Phys. Rev. B. 36 (1987) 8552.

[23] A. Crisanti and H.-J. Sommers, On The TAP Approach to the Spherical p-spin SG Model, preprint TNT-94-5, cond-mat/9406051, submitted to J. Phys. I France..

[24] R. Monasson and D. O'Kane, Domains of Solutions and Replica Symmetry Breaking in Multilayer Neural Networks, Europhys. Lett. 27 (1994) 85.

[25] A. J. Bray and M. A. Moore, Replica-Symmetry Breaking in Spin-Glass Theories, Phys. Rev. Lett. 41 (1978) 1068.

[26] A. J. Bray and M. A. Moore, Broken Replica Symmetry and Metastable States in Spin Glasses, J. Phys. C (Solid St. Phys.) 13 (1980) L907. 


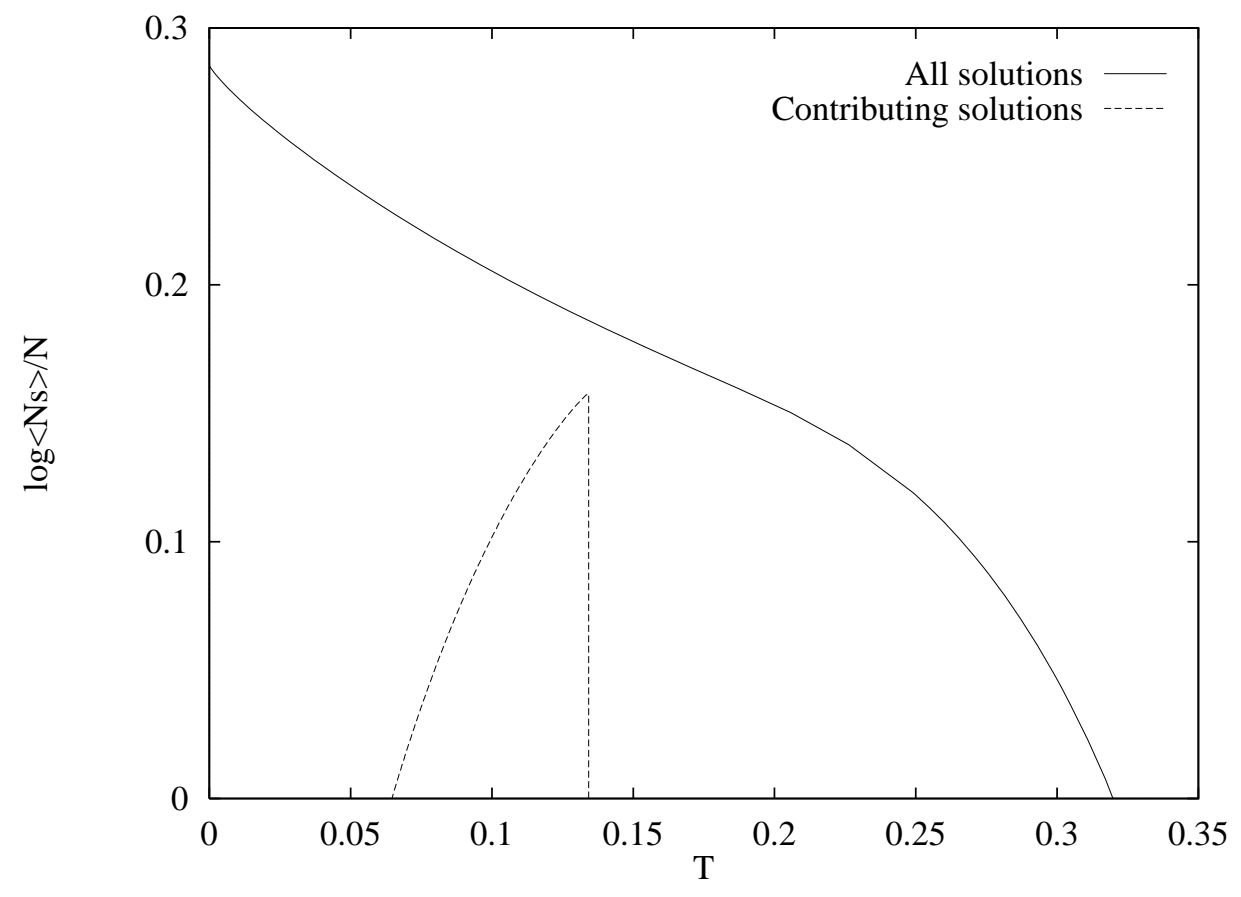

Figure 3: Normalized logarithm of the average total number of TAP solutions for ROM as a function of temperature $\left(N^{-1} \log \left\langle\mathcal{N}_{s}\right\rangle\right.$ vs $T$ ) (full line); same curve but for solutions that contribute to the free energy (dash line). 


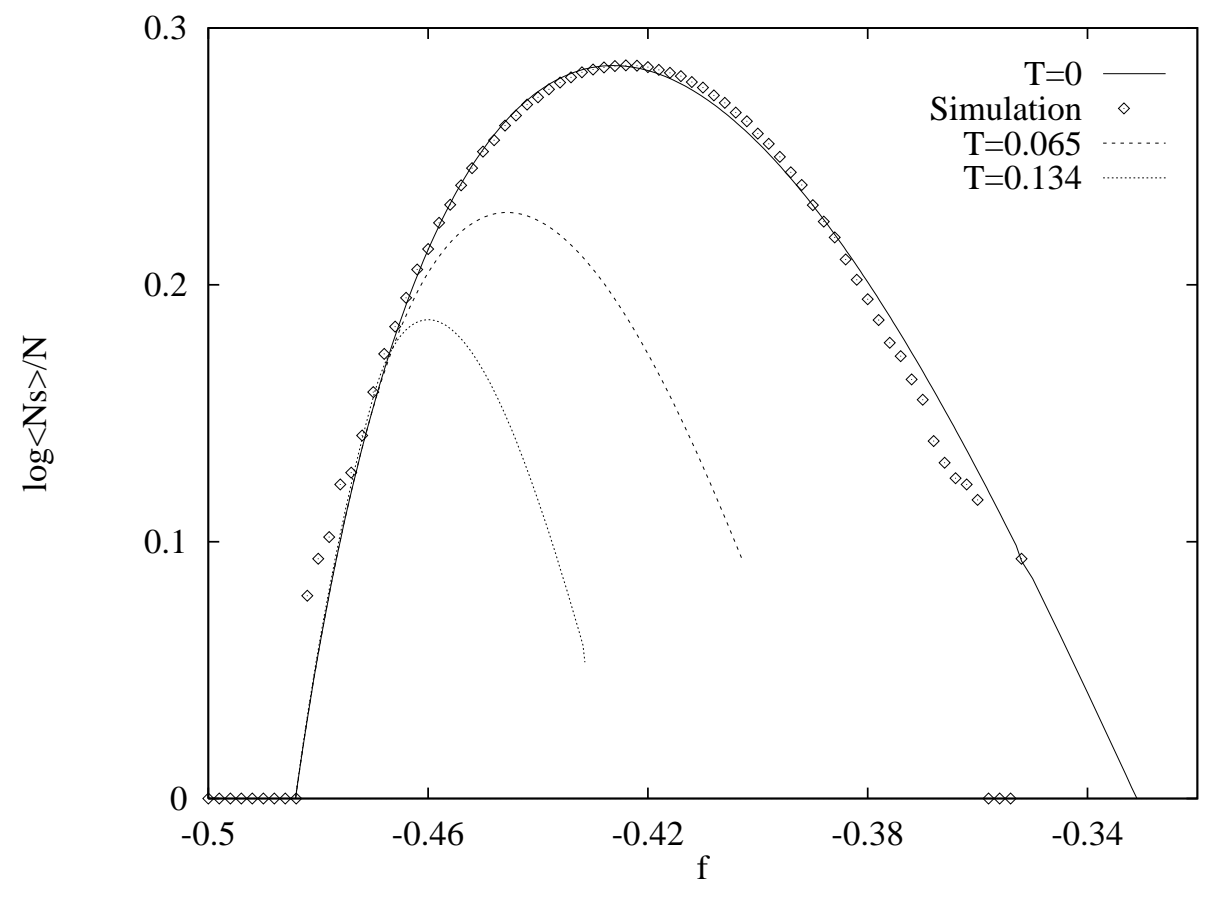

Figure 2: Logarithm of the density of metastable states divided by $N$ as a function of free energy $\left(N^{-1} \log \left\langle\mathcal{N}_{s}(f)\right\rangle\right.$ vs $f$ ) at $T=0$ (full line); $T=0.134$ (dash line) and $T=0.065$ (dotted line); Simulation at $T=0$ for $N=48$ Rom (data points). 


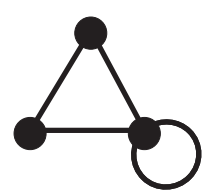

(a)

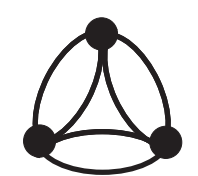

(b)

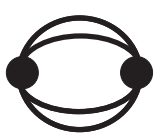

(c)

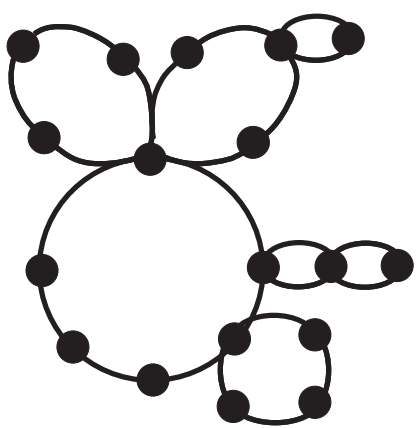

(d)

Figure 1: $(a)$ a diagram with a petal, $(b)$ and $(c)$ two non-cactus diagrams, $(d)$ a generic even-cactus diagram

$$
\begin{aligned}
& -\beta F(\beta)=N \log 2+\frac{\beta^{4}}{4} \sum_{i j k} J_{i j}^{2} J_{j k}^{2}+\frac{\beta^{4}}{12} \sum_{i j} J_{i j}^{4}+\frac{\beta^{4}}{8} \sum_{i j k l} J_{i j} J_{j k} J_{k l} J_{l i}+O\left(\beta^{5}\right) \\
& =N \log 2+\frac{\beta^{2}}{4} \sum_{i j} J_{i j}^{2} J_{j k} J_{k i}
\end{aligned}
$$

